\title{
Metal-Organic Framework as Catalyst in Esterification of Oleic Acid for Biodiesel Production
}

\author{
Kui Zhou and Somboon Chaemchuen
}

\begin{abstract}
Applying metal-organic frameworks (MOFs) as a solid catalyst in esterification of oleic acid and methanol for model of biodiesel production were investigated. Taking an advantage on high surface area, chemical/thermal stability and surface chemistry (acid/basicity) properties on MOF materials (MOF-808, ZIF-8, MIL-125) applied for catalyzed reaction of carboxylic acid to ester compound. Those MOF materials showed the catalytic properties higher than the reported alumina compound at same reaction condition. Their recyclability properties in heterogeneous catalyst were investigated. The characteristic properties of MOF materials such as crystal structure, porosity, surface area and functional group (chemical bonding) on spend catalyst after reaction were studied on this work.
\end{abstract}

Index Terms-Metal-organic frameworks, biodiesel, esterification, heterogeneous catalyst.

\section{INTRODUCTION}

Esterification plays an important with numerous application for industrial e.g. polymer, paints, varnishes, lacquers, medicines, dyes and synthetic rubber, including energy relative such as the production of biodiesel [1]. This reaction was also used to produce the bio-fuel or biodiesel from vegetable oil and/or animal fats. The product of biodiesel can utilized as fuel which their represent a safe, non-toxic and renewable energy source [2]. The catalyst is requirement to transformation the triglyceride (TGs) into fatty acid methyl esters (FAMEs) with ethanol. The source of biodiesel production are usually low cost feed stock such as waste cooking oils, by products of the vegetable oil refining, some non-edible oils, and animal fats etc., however, these source should contained free fatty acids (FFAs) less than $1 \%$ since an excess of FFAs causes side reaction (saponification) and problem in process of product purification (reduced the production yield and emulsion formation) [3], [4]. Whereas, the acid catalyst is very important for the industrial production since can convert FFAs to eater product and non-side reaction generated.

Generally, the most common catalyst widely used in biodiesel production are base catalyst such as $\mathrm{NaOH}$ or $\mathrm{KOH}$ etc. and acid catalyst such as $\mathrm{H}_{2} \mathrm{SO}_{4}$ or $\mathrm{HCl}$ etc., which those homogeneous catalyst have been proven as the most affective for acceleration reaction [5]. However, the homogeneous catalyst was several drawback for using in commercial scale in view of economic and environmental view point e.g. critical step to wash biodiesel product, waste of water and

Manuscript received September 2, 2016; revised November 11, 2016.

The authors are with Wuhan University of Technology, China (e-mail: 294435125@qq.com,sama_che@hotmail.com). water treatment, corrosive in metal devices of reactor and also increasing the cost of production, non-reusable catalyst and so on. Therefore, the developing in heterogeneous catalyst comes to replace with reducing the biodiesel production cost and more environmental friendly since the catalyst easy removable, non-corrosive, the recovery reused possible. During the last decades, several diverse heterogeneous catalyzed esterification have already been investigated. Some of the examples are ion-exchange resins having sulfonic acid groups [6], sulfated or mixed oxides [7], heteropoly acids [8], metal-containing molecular sieves [9], acid functionalized silica/mesoporous silica [10], carbonaceous acidic materials [11], zeolites [12] and so on. Moreover, the acidity combining or functionalized in high porosity in materials influenced to enhance the performance in catalytic activity [13], [14]. However, a serious drawbacks have been reported such as leaching of species in recyclability [15], high condition in term of temperature and reaction time on, the low diffusion of oil due to the small pore size, swelling tendency in solvent, low active site involve of low surface area etc [16]. On the basis of these considerations, the catalyst improvement of the esterification could represent one of the key points through which the whole process can result in being economically convenient.

High porosity materials like metal-organic frameworks (MOFs) which constructed framework from inorganic (metals cluster/nodes) with organic (ligand/linker) offer tremendous possibilities for catalyst application since their properties in high porosity, surface area, and enable for tailor design for tuning the acidity/basicity properties [17-19]. Here, we proposed to use MOFs which their high stability in chemical, physical and thermal properties in reaction condition to investigate on esterification. Further to understand the fundamental reasons, the oleic acid was used to study since there is a typically high content in feedstock oil of biodiesel production.

\section{Methodology}

\section{A. Synthesis MOFs}

The ZIF-8-SV synthesis was analogous as that reported elsewhere [1]. $0.42 \mathrm{~g}(1.606 \mathrm{mmol})$ of $\mathrm{Zn}\left(\mathrm{NO}_{3}\right) \cdot 4 \mathrm{H}_{2} \mathrm{O}$ (metal source) and $0.12 \mathrm{~g}(1.462 \mathrm{mmol})$ of 2 -methylimidazole (2-MIM) were dissolved in $32 \mathrm{ml}$ of DMF. The solution was transferred to a Teflon-lined stainless-steel autoclave $(35 \mathrm{ml})$ and heated in programmable oven at $5^{\circ} \mathrm{C} / \mathrm{min}$ to $140^{\circ} \mathrm{C}$ and held for $24 \mathrm{~h}$. The light yellow solid product was separated from mother liquor and washed with $N, N^{\prime}-$ dimethylformamide (DMF) three times, immersed in methanol for 3 days and dried at room temperature under 
vacuum for $12 \mathrm{~h}$. The dried material was transferred into a vacuum desiccator for further use.

The MOF-808 was synthesised using a conventional solvothermal heating method. A teflone liner $35 \mathrm{~mL}$ with $116.5 \mathrm{mg}(0.50 \mathrm{mmol})$ of $\mathrm{ZrCl}_{4}$ and $35.3 \mathrm{mg}(0.168 \mathrm{mmol})$ of 1,3,5-benzenetricarboxylic acid. Thereafter, $2.8 \mathrm{~mL}$ (49 $\mathrm{mmol}$ ) of acetic acid and $5 \mathrm{~mL}$ of DMF were introduced. The mixture was sonicated for an additional 20 minutes before being sealed and heated at $135{ }^{\circ} \mathrm{C}$ for a period of $24 \mathrm{~h}$. The mixture was subsequently cooled to room temperature in the oven before the precipitate was isolated by vacuum filtration and washed with DMF $(2 \times 30 \mathrm{~mL})$, acetone $(2 \times 30 \mathrm{~mL})$ and methanol $(3 \times 30 \mathrm{~mL})$. The resulting powder was dried in air.

The MIL-125 has been synthesized by a solvothermal method using a solvent mixture of DMF and methanol. In a typical synthesis, terephthalic acid $(7.6 \mathrm{mmol})$ and titanium isopropoxide ( $\mathrm{Ti}(\mathrm{O} i \mathrm{Pr}) 4,5.1 \mathrm{mmol})$ were dissolved in the mixture of DMF $(40 \mathrm{~mL})$ and dry methanol $(10 \mathrm{~mL}, 99.9 \%)$ and loaded into a three-neck round bottom flask $(100 \mathrm{~mL})$ equipped with a reflux condenser at $20^{\circ} \mathrm{C}$. The resulting mixture was crystallized by stirring under reflux conditions at $100{ }^{\circ} \mathrm{C}$ for $72 \mathrm{~h}$. After the reaction, the mixtures were allowed to cool down to room temperature, leading to the formation of a white crystalline product in the solution. It was further filtered and then washed with DMF. For further purification, the as-synthesized product was re-dispersed at $60^{\circ} \mathrm{C}$ in DMF for $2 \mathrm{~h}(100 \mathrm{~mL}$ of DMF per $1 \mathrm{~g}$ of product) and then in methanol for $2 \mathrm{~h}$ ( $100 \mathrm{~mL}$ of DMF per $1 \mathrm{~g}$ of product). Finally, it was dried overnight at $90^{\circ} \mathrm{C}$ under nitrogen.

\section{B. Characterizations}

Activation of the materials was done by in-situ evacuation under vacuum at $110^{\circ} \mathrm{C}$ overnight to obtain porous materials ready for the characterizations. The powder X-ray diffraction spectra (XRD) were collected using an Empyrean instrument from PANalytical applying a monochromatic $\mathrm{Cu} \mathrm{K}$ radiation at ambient conditions. The low-pressure gas adsorption isotherms were measured by the volumetric method on a Micromeritics instrument (ASAP2020). In all gas adsorption measurements 60-100 mg of sample was used. For the $\mathrm{N}_{2}$ gas adsorption analyses gases of purity $>99.999 \%$ were applied. Prior to the adsorption measurements, the samples were evacuated at $180^{\circ} \mathrm{C}$ under vacuum (initial activation) for about $3 \mathrm{~h}$. The micro pore surfaces were calculated by the Brunauer-Emmett-Teller (BET) and Langmuir method. The linearized BET and Langmuir equation were fit to the data within the range $0.02<P / P 0<0.2$.

\section{RESULTS AND DisCUSSION}

The alumina compound was considered first as catalyst in this work since their Lewis acid was reported for active side of trans/esterification reaction [20], [21]. Herein, the high stability on chemical (moisture, water and weak acid etc.) and temperature of several series in MOF materials were selected for alternative solid catalyst of oleic acid esterification. ZIF-8 is fist of MOFs which this work involved a comparative evaluation since there are previous studies to use as a catalyst in transesterification of vegetable oil [22]. Consequently, solid catalyst involved a comparative on this study of the catalyst activity in esterification of oleic acid with methanol, in all cases under the same operation condition. The different result in percentage yield of methyl oleate observed using the solid catalyst of MOFs comparable to meso-alumina and blank experiment (non-catalyst) as shown in Fig.1. The result present all MOFs catalyst obtained higher percentage of yield than meso-alumina catalyst at the same reaction condition.

Regarding recovery catalyst at the end of the esterification which is advantage of heterogeneous or solid catalyst, were determined on the MOFs catalyst. In view of high activity (\% yield of methyl oleate) obtaining on the first reaction test, afterward, the recycle experiment was obtained. The result on all the MOFs catalyst observed remain catalytic activity after second and third recycle experiment (the percentage yield on methyl oleate obtained closely or stable value). Furthermore, the weight of catalyst after reaction was evaluated after washed material with methanol and dried at $120^{\circ} \mathrm{C}$ under vacuum for $12 \mathrm{~h}$ before analysis. The MOF-808, MIL-125 and meso alumina were found weight loss less than $3 \%$ after third recycles of experiment reaction. Whereas weight loss more than $10 \%$ observed on ZIF-8 on every recycle reaction which represent in partial decomposed on this material during reaction.
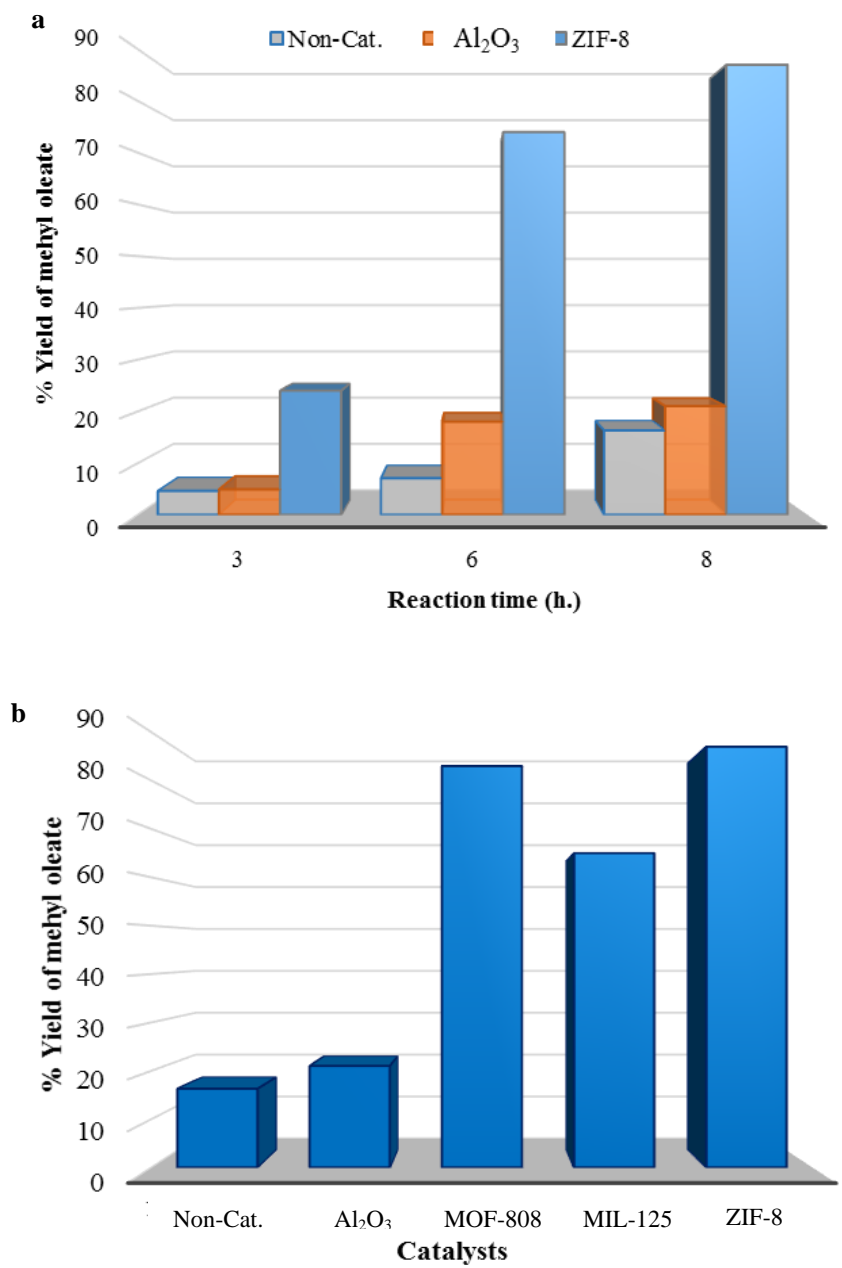

Fig. 1. The catalytic activity on Oleic acid esterification (\% Yield of methyl oleate) at $130^{\circ} \mathrm{C}, 0.1 \mathrm{~g}$ oleic acid, $1 \mathrm{ml}$ methanol and $10 \mathrm{mg}$ of catalyst; (a) the comparable $\%$ yield of Non-catalyst and reposted solid catalyst $\left(\mathrm{Al}_{2} \mathrm{O}_{3}\right.$ and ZIF-8) with time of reaction, and (b) the comparable activity in variance MOF catalyst after reaction $8 \mathrm{~h}$.

The properties of solid catalyst after reaction comparable to fresh sample were characterized such as the crystal 
structure (powerder X-ray diffraction), the porosity and surface area (gas adsorption, ASAP 2020) and chemical bonds or molecular structure (FT-IR) etc. The crystal structure of ZIF-8 after third recycle obtained similar as the fresh sample (Fig.2). However, the intensity was reduced which represent the lower in crystallinity of material. Whereas the absolutely change on XRD pattern was observed on MOF-808 and MIL-125 (Fig.2). Consequently, the analysis in chemical bonding or functional group of spend materials were investigated via FT-IR (Fig.3). The new spectrum (2800-2950 $\mathrm{cm}^{-1}$ ) were found on catalyst material after reaction. The $v_{\mathrm{O}-\mathrm{H}}$ stretch vibration band was reported a spectrum at $2500-3000 \mathrm{~cm}^{-1}$ which two of new spectrum (2845 and $2920 \mathrm{~cm}^{-1}$ ) were matching on $\mathrm{v}_{\mathrm{O}-\mathrm{H}}$ stretch vibration band [23]. Therefore, these result could evident that $\mathrm{O}-\mathrm{H}$ group in carboxylic group of oleic acid was bonding on solid catalyst during the reaction and still remain after reaction. Moreover, the reducing in porosity and surface area of materials after reaction also confirm that the carboxylic group (O-H group) of oleic acid coordinated and blocked the pore of material since spend catalysts showed non porous material. However, this results could not big effect to catalytic activity since the materials (MOFs) still shown remaining in catalytic activity (recycle ability).
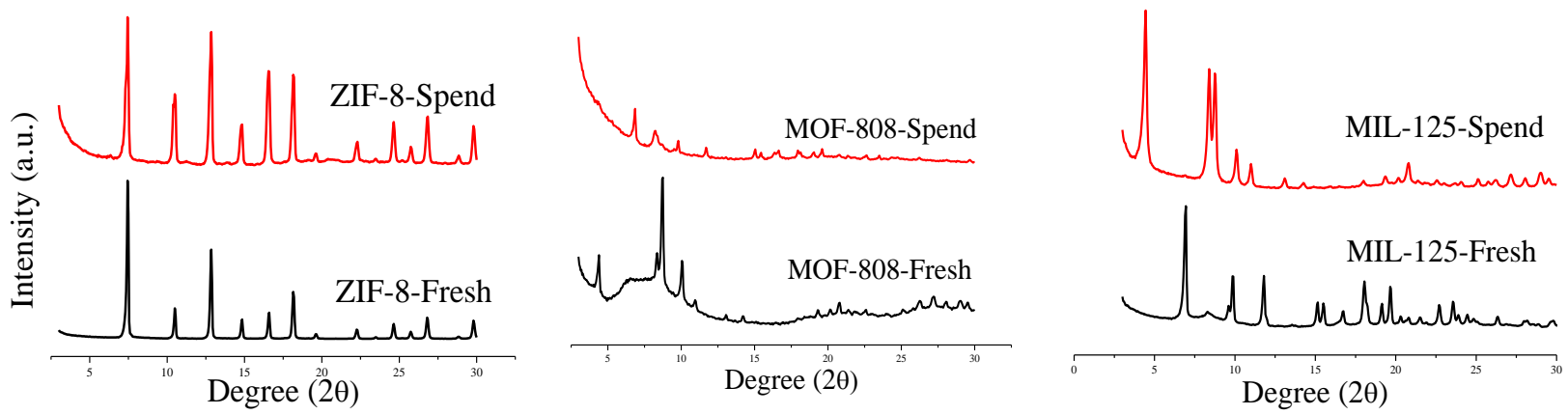

Fig. 2. The XRD pattern of MOFs on before (Fresh) and after (spend) catalytic reaction for $3^{\text {rd }}$ of reaction experiment.

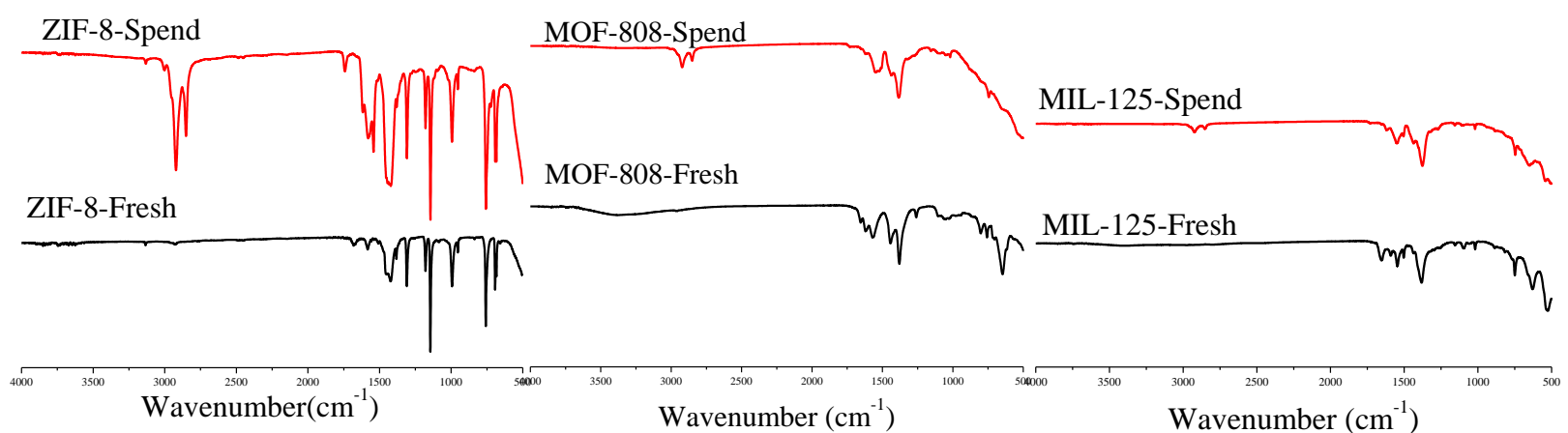

Fig. 3. The FT-IR spectrum of MOFs on before (Fresh) and after (Spend) catalytic reaction for $3^{\text {rd }}$ of reaction recycles.

\section{CONCLUSION}

The experimental addressing the oleic acid esterification using MOFs as a catalyst appears alternative for heterogeneous phase/solid catalyst which simple separation process. Moreover, those materials have ability to recycle catalyzed reaction in several times which high potential to use for catalyzed for biodiesel product in up-scale or industrial in further. The opportunity making biodiesel as the alternative fuel competition with traditional fossils fuel, the decreasing process cycle and costs is essential aspect in the biodiesel production. However, these material still required at high temperature which there are big challenging to improve or functionalize materials (MOFs) to achieve in requirement in industrial.

\section{ACKNOWLEDGMENT}

The authors are grateful to the State Key Lab of Advanced Technology for Materials Synthesis and Processing for financial support (Wuhan University of Technology). S.C. appreciates the National Natural Science Foundation of China (No. 201502146), The Fundamental Research Funds for the Central Universities (WUT: 2016IVA092) and the Research Fund for the Doctoral Program of Higher Education of China (471-40120222).

\section{REFERENCES}

[1] J. Otera and J. Nishikido, "Industrial Uses," Esterification: Methods, Reactions, and Applications, Second Edition, pp. 293-322.

[2] J. V. Gerpen, "Biodiesel processing and production," Fuel Processing Technology, vol. 86 pp. 1097-1107, 2005.

[3] J. F. Costa, M. F. Almeida, M. C. M. Alvim-Ferraz, and J. M. Dias, "Biodiesel production using oil from fish canning industry wastes," Energy Conversion and Management, vol. 74, pp. 17-23, 2013.

[4] Y. Jiang, J. Lu, K. Sun, L. Ma, and J. Ding, "Esterification of oleic acid with ethanol catalyzed by sulfonated cation exchange resin: Experimental and kinetic studies," Energy Conversion and Management, vol. 76, pp. 980-985, 2013.

[5] R. Tesser, M. D. Serio, M. Guida, M. Nastasi, and E. Santacesaria, "Kinetics of oleic acid esterification with methanol in the presence of triglycerides," Industrial \& Engineering Chemistry Research, vol. 44, pp. 7978-7982, 2005.

[6] M. A. Harmer and Q. Sun, "Solid acid catalysis using ion-exchange resins," Applied Catalysis A: General, vol. 221, pp. 45-62, 2001.

[7] N. A. Khan, D. K. Mishra, I. Ahmed, J. W. Yoon, J.-S. Hwang, and S. H. Jhung, "Liquid-phase dehydration of sorbitol to isosorbide using sulfated zirconia as a solid acid catalyst," Applied Catalysis A: General, vol. 452, pp. 34-38, 2013.

[8] M. N. Timofeeva, "Acid catalysis by heteropoly acids," Applied Catalysis A: General, vol. 256, pp. 19-35, 2003. 
[9] M. N. Timofeeva, V. N. Panchenko, Z. Hasan, N. A. Khan, M. S. Mel'gunov, A. A. Abel, M. M. Matrosova, K. P. Volcho, and S. H. Jhung, "Effect of iron content on selectivity in isomerization of $\alpha$-pinene oxide to campholenic aldehyde over Fe-MMM-2 and Fe-VSB-5," Applied Catalysis A: General, vol. 469, pp. 427-433, 2014.

[10] Z. Hasan and S. H. Jhung, "Facile in situ syntheses of highly water-stable acidic sulfonated mesoporous silica without surfactant or template," European Journal of Inorganic Chemistry, vol. 2014, pp. 3420-3426, 2014.

[11] A. Aldana-Pérez, L. Lartundo-Rojas, R. Gómez, and M. E. Niño-Gómez, "Sulfonic groups anchored on mesoporous carbon Starbons-300 and its use for the esterification of oleic acid," Fuel, vol. 100, pp. 128-138, 2012.

[12] G. Zhang, X. Zhang, J. Lv, H. Liu, J. Qiu, and K. L. Yeung, "Zeolite capillary microreactor by flow synthesis method," Catalysis Today, vol. 193, pp. 221-225, 2012.

[13] Z. Gao, S. Tang, X. Cui, S. Tian, and M. Zhang, "Efficient mesoporous carbon-based solid catalyst for the esterification of oleic acid," Fuel, vol. 140, pp. 669-676, 2015.

[14] S. S. Vieira, Z. M. Magriotis, M. F. Ribeiro, I. Graça, A. Fernandes, J. M. F. M. Lopes, S. M. Coelho, N. A. V. Santos, and A. A. Saczk, "Use of HZSM-5 modified with citric acid as acid heterogeneous catalyst for biodiesel production via esterification of oleic acid," Microporous and Mesoporous Materials, vol. 201, pp. 160-168, 2015.

[15] C. M. Garcia, S. Teixeira, L. L. Marciniuk, and U. Schuchardt, "Transesterification of soybean oil catalyzed by sulfated zirconia," Bioresource Technology, vol. 99, pp. 6608-6613, 2008.

[16] B. M. Reddy and M. K. Patil, "Organic syntheses and transformations catalyzed by sulfated zirconia," Chemical Reviews, vol. 109, pp. 2185-2208, 2009.

[17] S. Chaemchuen, K. Zhou, N. A. Kabir, Y. Chen, X. Ke, G. Van Tendeloo, and F. Verpoort, "Tuning metal sites of DABCO MOF for gas purification at ambient conditions," Microporous and Mesoporous Materials, vol. 201, pp. 277-285, 2015.

[18] S. Chaemchuen, N. A. Kabir, K. Zhou, and F. Verpoort, "Metal-organic frameworks for upgrading biogas via $\mathrm{CO} 2$ adsorption to biogas green energy," Chemical Society Reviews, vol. 42, pp. 9304-9332, 2013.

[19] B. Mousavi, S. Chaemchuen, B. Moosavi, Z. Luo, N. Gholampour, and F. Verpoort, "Zeolitic imidazole framework-67 as an efficient heterogeneous catalyst for the conversion of $\mathrm{CO} 2$ to cyclic carbonates," New Journal of Chemistry, 2016.
[20] D. Meloni, D. Perra, R. Monaci, M. G. Cutrufello, E. Rombi, and I. Ferino, "Transesterification of Jatropha curcas oil and soybean oil on Al-SBA-15 catalysts," Applied Catalysis B: Environmental, vol. 184, pp. 163-173, 2016

[21] G. X. Yu, X. L. Zhou, C. L. Li, L. F. Chen, and J. A. Wang, "Esterification over rare earth oxide and alumina promoted SO42-/ZrO2," Catalysis Today, vol. 148, pp. 169-173, 2009.

[22] C. Chizallet, S. Lazare, D. Bazer-Bachi, F. Bonnier, V. Lecocq, E. Soyer, A.-A. Quoineaud, and N. Bats, "Catalysis of transesterification by a nonfunctionalized metal- organic framework: acido-basicity at the external surface of ZIF-8 probed by FTIR and ab initio calculations," Journal of the American Chemical Society, vol. 132, pp. 12365-12377, 2010.

[23] N. P. Roeges, A Guide to the Complete Interpretation of Infrared Spectra of Organic Structures, Wiley, 1994.

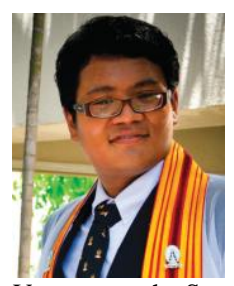

Somboon Chaemchuen was born in 1984, in Thailand. He received his $\mathrm{PhD}$ in chemical engineering from Chulalongkorn University, Thailand, in 2011 under the supervision of Dr Suphot Phatanasri. In 2010, he went to Aachen University, Germany, for research collaboration with Prof. Wolfgang F. Holderich. He has recently obtained an assistant researcher position in the group of Prof. Francis Verpoort at the State Key Laboratory of Advanced Technology for Materials Synthesis and Processing, Wuhan University of Technology. His current research interests are the preparation of metal-organic framework (MOF) compounds and the study of their applications.

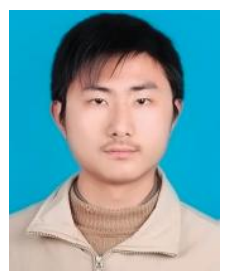

Kui Zhou was born in1990, in China. He received his bachelor degree in material science \& technology (Wuhan University of Technology, China) in 2012. He is currently a master student under the supervision of Prof. Francis Verpoort from the Laboratory of Organometallics, Catalysis and Ordered Materials (LOCOM), State Key Laboratory of Advanced Technology for Materials Synthesis and Processing, Wuhan University of Technology. His current research interests are in synthesis and modification of metal-organic frameworks (MOFs). 